\title{
Panorama da Última Década de Pesquisas com Testes de Consciência Fonológica
}

\author{
Dalva Maria Alves Godoy ${ }^{1}$ \\ Natália Fortunato \\ Aline Paiano \\ Departamento de Pedagogia da Universidade do Estado de Santa Catarina, Florianópolis, \\ Santa Catarina, Brasil
}

\begin{abstract}
Resumo
A consciência fonológica se constitui em um conjunto de habilidades que tem sido evidenciado como diretamente relacionado à aprendizagem da leitura e da escrita em diferentes línguas e ortografias. O presente artigo objetiva desenhar um panorama das pesquisas brasileiras no período entre 2000 e 2010 que investigaram essas habilidades metalinguísticas com a aplicação de testes. A amostra, obtida a partir do portal CAPES de teses e dissertações, foi analisada sob diversos aspectos: por área de formação inicial e em nível de pós-graduação dos pesquisadores; tipo de teste de consciência fonológica utilizado; tema investigado e tipo, característica e faixa de escolarização da população investigada. Além disso, deu-se especial atenção aos estudos longitudinais, de treinamento e com sujeitos disléxicos, com a finalidade de evidenciar os principais resultados. A análise mostra concentração de estudos pelos profissionais da área da saúde com temática relacionada à leitura e escrita, em população de crianças sem queixa de dificuldades de aprendizagem. Os estudos longitudinais e, principalmente, os de treinamento evidenciaram o caráter crucial da consciência fonológica para a aprendizagem alfabética. As pesquisas com disléxicos, ainda que em número inexpressivo, apontaram para a pertinência do prejuízo em consciência fonológica. Esses resultados confirmam a importância das habilidades de consciência fonológica para a aprendizagem da leitura e da escrita no português do Brasil, como também revelam aspectos a serem mais bem aprofundados e discutidos.
\end{abstract}

Palavras-chave: Consciência fonológica, aprendizagem da leitura e da escrita, dislexia.

\section{Overview of the Last Decade of Research with Tests of Phonological Awareness}

\begin{abstract}
Phonological awareness constitutes a set of skills that have been shown to be directly related to the learning of reading and writing in different languages and orthographies. This paper aims to draw a panorama of Brazilian research that investigated these metalinguistic skills through the application of tests between the years 2000 and 2010. The sample, obtained from the portal of theses and dissertations of CAPES, was analyzed in several ways: by area of initial formation and the postgraduate level of the researchers; type of phonological awareness test used; theme of investigation, and type, features and range of schooling of the population investigated. Furthermore, special attention was given to longitu-
\end{abstract}

Endereço para correspondência: Departamento de Pedagogia, Centro de Ciências da Educação, Universidade do Estado de Santa Catarina, Av. Madre Benvenutta, 2007, Itacorubi, Florianópolis, SC, Brasil 88035-001. E-mail: dalvagodoy@gmail.com,natipedago@gmail.com e alinepaiano1@hotmail.com 
dinal studies, training ones and ones with dyslexic subjects, in order to highlight the main results. The analysis shows the concentration of studies by professionals in the field of health with themes related to reading and writing in a population of children with no complaint of learning disabilities. Longitudinal studies, and especially the training ones showed the crucial nature of phonological awareness to alphabet learning. Research with dyslexics though unimpressive in number, pointed to the relevance of the damage in phonological awareness. These results confirm the importance of the skills of phonological awareness to the learning of reading and writing in Brazilian Portuguese, while indicating aspects to be better investigated and discussed.

Keywords: Phonological awareness, learning of reading and writing, dyslexia.

\section{Panorama Acerca de la Investigación con Pruebas de Consciencia Fonológica en la Última Década}

\section{Resumen}

La conciencia fonológica constituye un conjunto de habilidades que han demostrado estar directamente relacionado con el aprendizaje de la lectura y la escritura en diferentes lenguas y ortografías. Este artículo pretende dibujar un panorama de los estudios brasileños que, entre 2000 y 2010, investigaron esas habilidades metalingüísticas por medio de aplicaciones de pruebas. La muestra, obtenida del portal CAPES de tesis y disertaciones, fue analizada en diferentes maneras: por área de grado y posgrado de los investigadores, el tipo de prueba de conciencia fonológica utilizado; tema investigado y el tipo, característica y escolarización de la población investigada. Además, se prestó especial atención a los estudios longitudinales, de entrenamiento y con disléxicos con el fin de conocer los principales resultados. El análisis pone de manifiesto la concentración de estudios ejecutados por profesionales de salud, con tema sobre la lectura y la escritura, con población de niños sin dificultades de aprendizaje. Los estudios longitudinales y en especial, los de entrenamiento, han demostrado la importancia crucial de la conciencia fonológica para el aprendizaje alfabético. La investigación con disléxicos, aunque en número inexpresivo, señaló la propiedad del prejuicio de la conciencia fonológica. Estos resultados confirman la importancia de la conciencia fonológica para aprender a leer y escribir en portugués de Brasil, pero también revelan aspectos que deben ser investigados y discutidos más a fondo.

Palabras clave: Conciencia fonológica, aprendizaje de la lectura y de la escritura, dislexia.

Nos últimos 40 anos houve um avanço expressivo nas investigações da área de leitura. A psicologia cognitiva, interessada em compreender quais os processos envolvidos no ato de ler e escrever, produziu inúmeras evidências sobre a importância da consciência fonológica para a aprendizagem alfabética e destacou a consciência fonêmica como uma habilidade estreitamente relacionada ao sucesso dessa aprendizagem (Castles \& Coltheart, 2004; Liberman, Shankweiler, Fischer, \& Carter, 1974).

Entende-se consciência fonológica como um conjunto de habilidades, integrante das habilidades metalinguísticas, que possibilita ao sujei- to refletir e manipular conscientemente os sons da fala (Scliar-Cabral, 2003). As habilidades de consciência fonológica, também denominadas de habilidades metafonológicas, podem ser mensuradas por meio de tarefas de identificação e de manipulação de partes sonoras das palavras, tais como a identificação de rimas, sílabas e fonemas e a manipulação de sílabas e de fonemas cujas tarefas podem exigir operações de análise e/ou síntese e de memória de trabalho. Segundo Stanovich, Cunningham e Cramer (1984), as tarefas que avaliam a consciência fonológica variam em graus de dificuldade. Assim, tarefas que exigem a manipulação explícita de unidades são mais di- 
fíceis do que tarefas de identificação, ao passo que tarefas em nível silábico são mais fáceis do que tarefas em nível fonêmico.

Desse conjunto de habilidades, a consciência fonológica no nível do fonema tem sido apontada como o mais forte fator preditivo de sucesso em leitura: bons níveis de consciência fonêmica, ao início do processo de alfabetização, produzem futuros bons leitores (Bradley \& Bryant, 1983; Cardoso-Martins, 1995; Defior \& Tudela, 1994; Jong \& Van Der Leij, 1999; Lundberg, Frost, \& Petersen, 1988; Porpodas, 1991; Wimmer, Landerl, Linortner, \& Hummer, 1991). Algumas habilidades metafonológicas, como a identificação de rimas e a consciência silábica, se desenvolvem no curso da experiência linguística; entretanto, a consciência fonêmica só é passível de se desenvolver quando o sujeito é exposto ao ensino do código alfabético (Bertelson \& Gelder, 1991; Morais, Cary, Alegria, \& Bertelson, 1979; Morais, Bertelson, Cary, \& Alegria, 1986; Read, Zhanc, Nie, \& Dincg, 1986). Esse aparente impasse gerou sérios confrontos, inclusive porque se entendeu que a consciência fonêmica era um pré-requisito para a alfabetização. De forma consensual, na atualidade, os pesquisadores admitem uma relação intrínseca e recíproca entre as habilidades de consciência fonológica e a aprendizagem da leitura, de tal modo que a presença de habilidades precoces, no nível da rima e da sílaba, facilita a aquisição da leitura, e o desenvolvimento desta última, propicia o desenvolvimento do nível fonêmico que, por sua vez, impulsiona a aprendizagem da leitura (Alegria, Leybaert, \& Mousty, 1997; Rayner \& Pollatsek, 1989).

Em função da importância dessas habilidades para a aprendizagem, assistiu-se a um crescente interesse por parte dos pesquisadores em compreender em que medida a consciência fonológica se relaciona aos aspectos de aquisição, desenvolvimento e reabilitação da linguagem oral, mas, sobretudo, da linguagem escrita. Um corpo considerável de pesquisas tem igualmente investigado qual a relação das habilidades metafonológicas com os distúrbios específicos de leitura, a dislexia, tendo constatado que um prejuízo nas habilidades de consciência fonêmica é a principal causa da dislexia (Jong, 2003; Rack, Snowling, \& Olson, 1992; Snowling, 1998).

Pesquisas mais recentes têm se debruçado em dimensionar a contribuição específica da consciência fonológica em função das características de cada ortografia. Nas ortografias transparentes, em que as relações grafofonológicas são guiadas em sua maioria por regras, o processo de decodificação é aprendido mais rapidamente, e com isso a influência da consciência fonológica pode estar restrita a um período menor de tempo, logo ao início do processo de alfabetização. Nas ortografias opacas, diferentemente, em função da inconsistência das correspondências grafofonológicas, a consciência fonológica é recrutada por um período maior de tempo (Aro \& Wimmer, 2003; Defior, 2004; Defior, Martos, \& Cary, 2002; Vaessen \& Blomert, 2010; Wimmer \& Goswami, 1994). Tomando-se em consideração as características de cada ortografia, as evidências já sedimentadas em relação à dislexia também têm sido revistas com a finalidade de entender melhor como essa dificuldade se manifesta nas diferentes ortografias (Landerl, Wimmer, \& Frith, 1997; Serrano \& Defior, 2008).

No Brasil, nos últimos 20 anos, aproximadamente, o tema ocupou a atenção de muitos pesquisadores. Segundo Maluf, Pagnez e Zanella (2006), grande parte da produção brasileira entre 1987 e 2005 que investigou a relação entre as diferentes habilidades metalinguísticas (lexical, morfológica, sintática, semântica, fonológica, ortográfica) e a aprendizagem da linguagem escrita elegeu como principal foco o estudo da consciência fonológica: 80 dos 113 trabalhos de teses e dissertações. No que diz respeito a instrumentos para a avaliação das habilidades fonológicas, já a partir dos anos 80 , encontramos registro de uma prova de segmentação fonêmica, utilizada por Carraher e Rego (1981), e um conjunto de quatro provas de consciência fonológica: segmentação, síntese e exclusão fonêmica e identificação de rimas, proposto por Fernandes, Andrade, Befi-Lopes e Wertzner (1998). Os primeiros testes publicados a que tivemos acesso, conforme Godoy (2001) foram os de Santos e Pereira (1997) e o de Capovilla e Capovilla (1998). Ambos tomaram como referência o teste 
de Hatcher (1994) e o teste de Capovilla e Capovilla (1998) menciona também ter se baseado no de Santos e Pereira (1997). A partir daí muitos pesquisadores passaram a construir suas próprias tarefas para avaliar um ou outro nível de consciência fonológica, como também alguns tomaram a iniciativa de construir ou adaptar baterias de testes. Como resultado, muitos trabalhos de investigação pautaram-se na utilização de um ou de outro teste desenvolvido por autores diversos, sem haver, entretanto, um teste de referência padronizado. Ressalta-se que o teste de Capovilla e Capovilla (1998) objetivou estabelecer a normatização dos resultados para a faixa etária de 3 a 8 anos, o que, segundo os autores, poderia ser útil para o treino preventivo e remediativo dos problemas de aquisição e desenvolvimento da linguagem escrita (Capovilla, Capovilla, \& Silveira, 1998).

Considerando esse movimento, este artigo objetiva desenhar um panorama da pesquisa nacional dos últimos dez anos (2000 a 2010) que fez uso de testes de consciência fonológica. Em primeiro lugar buscou-se verificar quantos e quais foram os testes de avaliação da consciência fonológica publicados e utilizados pelos pesquisadores e, a partir desse levantamento, tencionou-se desenhar um panorama em relação às temáticas, o tipo de população e a faixa de escolarização investigadas pelas pesquisas empíricas no período, bem como quantas pesquisas se dedicaram a estudar a população de sujeitos com dificuldades de aprendizagem ${ }^{2}$ e que resultados suportam em relação à participação das habilidades de consciência fonológica.

Especial atenção foi dada aos estudos longitudinais e de treinamento, pois, como afirma Alegria et al. (1997, p. 120), a única "maneira

2 Em função da diversidade de termos utilizados pelos pesquisadores para denominar as dificuldades em aprender a ler e/ou a escrever, neste artigo o termo "dificuldade de aprendizagem" engloba: dificuldade de aprendizagem, de leitura, de linguagem escrita, de codificação e decodificação do fonema, como também distúrbio de aprendizagem e dislexia. indiscutível" de determinar o sentido causal entre duas variáveis relacionadas, no caso, a consciência fonológica e a aprendizagem da leitura e da escrita, é realizar estudos longitudinais em que são propostos treinamentos de uma variável, considerada causal, e observado o comportamento da variável tida como efeito, em um momento posterior ${ }^{3}$. Desse modo, os resultados de estudos longitudinais e de treinamento em consciência fonológica foram analisados a fim de ressaltar as evidências do papel da consciência fonológica em relação ao aprendizado da leitura. De certo modo, a intenção em traçar esse panorama é a de construir um ensaio sobre o estado da arte no momento atual no cenário brasileiro. Com isso espera-se contribuir para nortear futuras pesquisas na área.

\section{Método}

A amostra foi selecionada a partir da base de dados da CAPES de teses e dissertações (último acesso em 30 de novembro de 2011). A busca foi realizada com os seguintes termos: "consciência fonológica" e "consciência fonêmica", todos os índices, todas as fontes; "testes de consciência fonológica”, expressão exata; restritos ao período alvo da investigação. O foco sobre as habilidades de consciência fonológica em detrimento às demais habilidades metalinguísticas se justi-

3 Nos estudos longitudinais são observadas as correlações entre variável critério, ou dependente, e variável independente. A variabilidade da variável dependente pode ser predita pela variabilidade da variável independente (Correlação de Pearson). Como exemplo, sabe-se que a habilidade em leitura é fortemente influenciada pela habilidade de consciência fonêmica. Assim, para se determinar o efeito causal da consciência fonêmica sobre a leitura, o estudo promove o treino em consciência fonêmica em um momento inicial e observa, em um momento posterior, o efeito desse treinamento sobre os desempenhos em leitura. Esse desenho de pesquisa sempre utiliza o controle de todas as variáveis envolvidas no processo, em todos os momentos de testagem. Assim, o controle das variáveis de consciência fonêmica e leitura é feito no início e no final do experimento. 
fica em função da larga evidência na literatura ao enfatizar esta habilidade como fundamental para a aquisição do processo de decodificação e compreensão.

Procedeu-se à leitura dos resultados preliminares, captados em forma de resumo, com o objetivo de selecionar apenas os trabalhos empíricos com avaliação da consciência fonológica. Nos casos em que no resumo não foi identificado qual teste foi utilizado, procedeu-se à busca do trabalho na íntegra através de sites da internet ou mesmo procurou-se fazer contato pessoal com o autor a fim de obter o trabalho.

A amostra foi analisada e distribuída por ano de publicação, por área de formação inicial dos autores e subsequente área de concentração do programa de pós-graduação em que foi defendida a dissertação ou tese. Em seguida foi classificada conforme o teste utilizado pelo pesquisador. Uma vez que o objetivo deste estudo foi analisar apenas os trabalhos empíricos em consciência fonológica, quando o trabalho não informava qual teste havia sido utilizado, este foi retirado das análises subsequentes.

A amostra resultante, portanto, foi analisada segundo o tema investigado, tipo e características da população e faixa de escolarização. Com relação à faixa de escolarização, foram estabelecidas, inicialmente, as seguintes categorias: pré-escolar, $1^{\mathrm{a}}$ a $4^{\mathrm{a}}$ série, $5^{\mathrm{a}}$ a $8^{\mathrm{a}}$ série, ensino médio e EJA - Educação de Jovens e Adultos de $1^{\mathrm{a}}$ a $4^{\text {a }}$ série. No entanto, muitos trabalhos tiveram como alvo populações que transbordavam essas faixas, e foi preciso estabelecer faixas mais amplas. A análise dos trabalhos seguiu, portanto, as seguintes categorias:

- Tema investigado: leitura e escrita (1), linguagem oral (2), consciência fonológica pura (3);

- Tipo de população: crianças (1), adultos (2), comparação entre crianças e adultos (3);

- Características da população: sem queixa de dificuldades (1), com queixa de dificuldades (2), comparação de sujeitos com e sem queixa de dificuldades (3);

- Faixa de escolarização: pré-escolar (1), $1^{\text {a a }}$ $4^{\text {a }}$ série (2), pré-escolar a $4^{\mathrm{a}}$ série (3), $1^{\mathrm{a}}$ a $8^{\mathrm{a}}$ série (4), EJA - $1^{\mathrm{a}}$ a $4^{\mathrm{a}}$ série (5), sem especificação (6): quando não havia informação compatível.

Com a finalidade de ratificar resultados em relação à hipótese causal entre consciência fonológica e aprendizagem da leitura, foram selecionados os trabalhos caracterizados por estudos longitudinais e de treinamento para análise. Nos resultados dos estudos longitudinais interessava colocar em evidência quais as conclusões sobre a qualidade da relação entre consciência fonológica e aprendizagem da leitura. Assim, os resultados foram categorizados como: "não há relação", "há relação", "há relação recíproca", "há relação direta e preditiva", "não estudou a relação". Já nos estudos que treinaram a consciência fonológica, interessava colocar em evidência quais os resultados obtidos com o treino realizado: se houve ou não melhora da aprendizagem da leitura.

Além dessas análises, para verificar como os pesquisadores relacionam as dificuldades de aprendizagem às habilidades de consciência fonológica, selecionaram-se os trabalhos em que, nos resumos e palavras-chave, houvesse os termos "dislexia", "dificuldade", "distúrbio", e posteriormente os resultados descritos nesses trabalhos foram analisados com o intuito de reunir as principais evidências com relação à contribuição das habilidades de consciência fonológica aos fenômenos.

Para atender ao objetivo principal do presente estudo, os dados foram submetidos apenas a análise estatística descritiva com o intuito de desenhar o panorama das pesquisas, em âmbito nacional, que se utilizaram de testes de consciência fonológica entre os anos de 2000 a 2010.

\section{Resultados}

O levantamento inicial realizado junto ao portal CAPES de teses e dissertações resultou em 125 trabalhos que fizeram uso de testes de consciência fonológica em suas investigações. A Figura 1 mostra a distribuição desses trabalhos no decorrer da última década. 


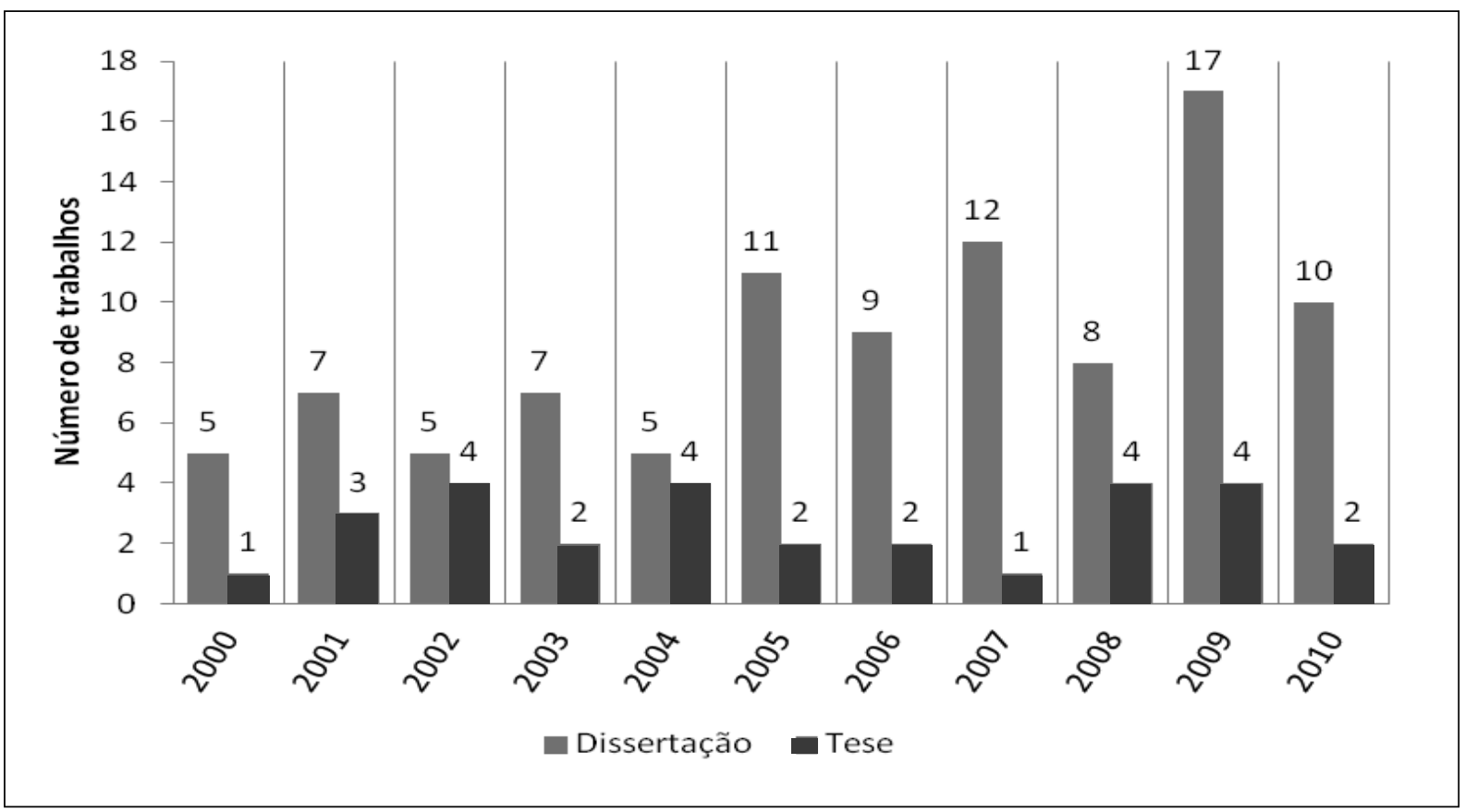

Figura 1. Distribuição das pesquisas no período entre 2000 a 2010, por ano e tipo de pesquisa (dissertação ou tese).

O número de dissertações (96) é muito maior do que os trabalhos de tese (29). Considerando que as pesquisas em nível de doutoramento devem apresentar contribuição inédita para o conhecimento científico, além de exaustiva pesquisa sobre métodos e teorias a respeito da temática em foco, podemos inferir que, ainda que sejam numerosas as pesquisas em relação à consciência fonológica, ela deve ser mais bem discutida e aprofundada no futuro.

A área de formação inicial do pesquisador, bem como a área do programa de pós-graduação em que se desenvolveram essas pesquisas, é apresentada na Tabela 1.

\section{Tabela 1}

\section{Distribuição das Pesquisas segundo a Área de Graduação e Pós Graduação}

\begin{tabular}{lcccc}
\hline & \multicolumn{3}{c}{ Área de conhecimento } \\
\cline { 2 - 4 } Graduação & \multicolumn{3}{c}{ Pós-graduação } & \multirow{2}{*}{ Totais } \\
\cline { 2 - 5 } & Ciências da Saúde & Linguística, Letras e Artes & Ciências Humanas & \\
\hline Fonoaudiologia & 37 & 21 & 15 & 73 \\
Psicologia & - & - & 26 & 26 \\
Pedagogia & - & 1 & 17 & 18 \\
Letras & - & 5 & 1 & 6 \\
Artes & - & - & 1 & 1 \\
Música & - & 1 & - & 120 \\
\hline Totais & 37 & 28 & 60 & 125 \\
\hline
\end{tabular}

Nota. Valores correspondem ao número de trabalhos realizados no período entre 2000 a 2010, segundo a área de graduação do pesquisador e área de concentração de conhecimento do programa de pós-graduação. Fonte: dados da pesquisa. 
Destaca-se o interesse dos profissionais ligados à clínica de reabilitação de distúrbios de linguagem, como fonoaudiólogos e psicólogos, por compreenderem a contribuição da consciência fonológica para esses fenômenos. Esses profissionais buscam aprofundar sua formação nas áreas das ciências humanas, da saúde e da linguística. É expressivo também, ainda que em menor número, o interesse dos profissionais ligados diretamente ao ensino, como pedagogos e professores de letras, por investigarem o tema, buscando formação complementar principalmente na área das ciências humanas.

Podemos inferir com isso que, apesar de as habilidades de consciência fonológica estarem implicadas na descoberta do princípio alfabético e serem habilidades recomendadas como facilitadoras do processo de aquisição da leitura e da escrita, ainda é fraca a inserção deste construto teórico na área diretamente ligada ao ensino. Fica para os profissionais empenhados na reabilitação dos distúrbios o emprego dessa habilidade como forma de restabelecer o curso da aprendizagem alfabética. Sem dúvida, parece haver uma lacuna teórico-metodológica, que poderá ser alcançada com a aproximação dos professores à produção e divulgação de pesquisas no campo educacional, mas que ao mesmo tempo, depende da aplicação de diretrizes curriculares e políticas públicas.

Os trabalhos recolhidos foram distribuídos segundo o teste de consciência fonológica utilizado pelo pesquisador. Os testes, em sua maioria, foram alcunhados pelo(s) nome(s) do(s) autor(es). Atendendo aos objetivos de pesquisa, dos 125 trabalhos inicialmente coletados, 32 não puderam ser analisados, pois em seus resumos não havia identificação do teste de consciência fonológica utilizado pelo pesquisador e, apesar dos esforços, o trabalho na íntegra não foi encontrado. Com isso, a amostra final analisada resultou em 93 trabalhos. A Tabela 2 mostra a quantidade de trabalhos que utilizaram cada um dos testes encontrados.

Nesse cenário, chama a atenção a eleição dos pesquisadores por dois testes: Capovilla e Capovilla $(1998,2000)$ e Confias (Moojen et
Tabela 2

Quantidade de Dissertações/Teses que Aplicaram cada Teste

Teste de consciência fonológica Quantidade

de trabalhos

1. Capovilla e Capovilla (1998 e 2000) 26

2. Confias (Moojen et al., 2003) 20

3. Cielo (2002) 9

4. Cardoso-Martins (1991) e adaptações 6

5. Santos e Pereira (1997) 5

6. Metafonológico sequencial (1991)* 3

7. Cognitivo Linguistico/Protocolo 3 de Capellini e Smythe (2008)*

8. Alvarez, Carvalho e Caetano 3 (1998 e 2004)

9. $\mathrm{LAC}^{* *}$ е CTOPP*** $(2002)^{*} \quad 2$

10. Herrero $(2001)^{*} 2$

11. BALESC**** $(2001)^{*} 2$

12. Diniz $(2008)^{*}$

13. Pacheco (2009)* 1

14. Alencar $(2000)^{*} \quad 1$

15. Aquino (2007)* 1

16. Corrêa $(2009)^{*} \quad 1$

17. Guedes $(2009)^{*} \quad 1$

18. Santos $(1996)^{*}$

19. Siccherino $(2007)^{*} \quad 1$

20. Carraher e Rego (1981)* 1

21. Fernandes et al. (1998)* 1

22. Barrera $(2000)^{*} \quad 1$

23. Ilha***** (2004)* 1

Total 93

Nota. Testes utilizados nas pesquisas brasileiras no período de 2000 a 2010 e quantidade de dissertações/teses que aplicaram cada teste. Fonte: dados da pesquisa.

*Construção de teste para avaliação de consciência fonológica pelo próprio autor da dissertação/tese ou adaptação de um teste já existente. **LAC - Lindamood Auditory Conceptualization Test (Lindamood \& Lindamood, 1979). ***CTOPP - Comprehensive Test of Phonological Processing (Wagner, Torgesen, \& Rashotte 1999). ****BALESC - Bateria de Avaliação da Linguagem Escrita e seus Distúrbios. Adaptação da BELEC - Batterie d'évaluation du langage écrit et de ses troubles (Mousty, Leybaert, Alégria, Content, \& Morais, 1994). *****Este trabalho, em função de ter utilizado como referência a adaptação de parte do teste de Capovilla e Capovilla (2000) e do de Cardoso-Martins (1991), foi contabilizado como um novo teste. 
al., 2003). Em conjunto, as pesquisas que utilizaram esses testes totalizaram $49 \%$ da produção nacional no período. Entretanto, é também relevante a quantidade de testes e/ou tarefas de consciência fonológica que foram elaborados/ adaptados e utilizados tão somente pelo autor do trabalho. Essa diversidade de testes e de tarefas que os compõem torna difícil a comparação de resultados obtidos tanto em relação à participação da consciência fonológica no processo de aprendizagem da leitura e da escrita como também para que se possa estabelecer como a consciência fonológica se desenvolve. Este aspecto foi, sem dúvida, um fator de limitação para a análise deste estudo. Como enfatizou Stanovich et al. (1984), uma criança pode apresentar desempenho satisfatório em uma tarefa fonológica e fracasso total em outra, dependendo do nível de dificuldade de cada tarefa. O conjunto de habilidades fonológicas, como mencionado, é mensurado por diferentes tarefas que exigem diferentes operações de análise e/ou síntese e de memória de trabalho. Além disso, algumas tarefas de consciência fonológica propostas/adaptadas pelos próprios pesquisadores carecem de confiabilidade, pois ao intencionarem medir essa habilidade estão, na verdade, medindo a representação ortográfica, ao introduzirem, por exemplo, letras como apoio, ou almejando, em tarefas de inversão de fonemas em palavras, como por exemplo: "amor" /amor/, uma resposta como "Roma" /Rome/, deixando com isso transparecer total desconhecimento sobre a representação fonológica dos segmentos (Godoy, Koglin, \& Abreu, 2011). Quando as pesquisas investigam a habilidade de consciência fonológica com instrumentos frágeis, sem rigor metodológico, com diversidade de tarefas que medem diferentes aspectos desta habilidade e em diferentes níveis, não é possível reunir conclusões confiáveis.

Com relação ao eixo temático de que trata os 93 trabalhos, 59 (63\%) se dedicaram a relacionar a habilidade de consciência fonológica à aprendizagem e/ou desenvolvimento e/ou dificuldades da leitura e da escrita. Onze trabalhos $(12 \%)$ relacionaram essa habilidade à aquisição ou dificuldade de aquisição e/ou desenvolvimento da linguagem oral. Finalmente, 23 trabalhos (25\%) estudaram a Consciência Fonológica como construto $(\mathrm{Mo}=1)$. Estes resultados estão ilustrados na Figura 2.

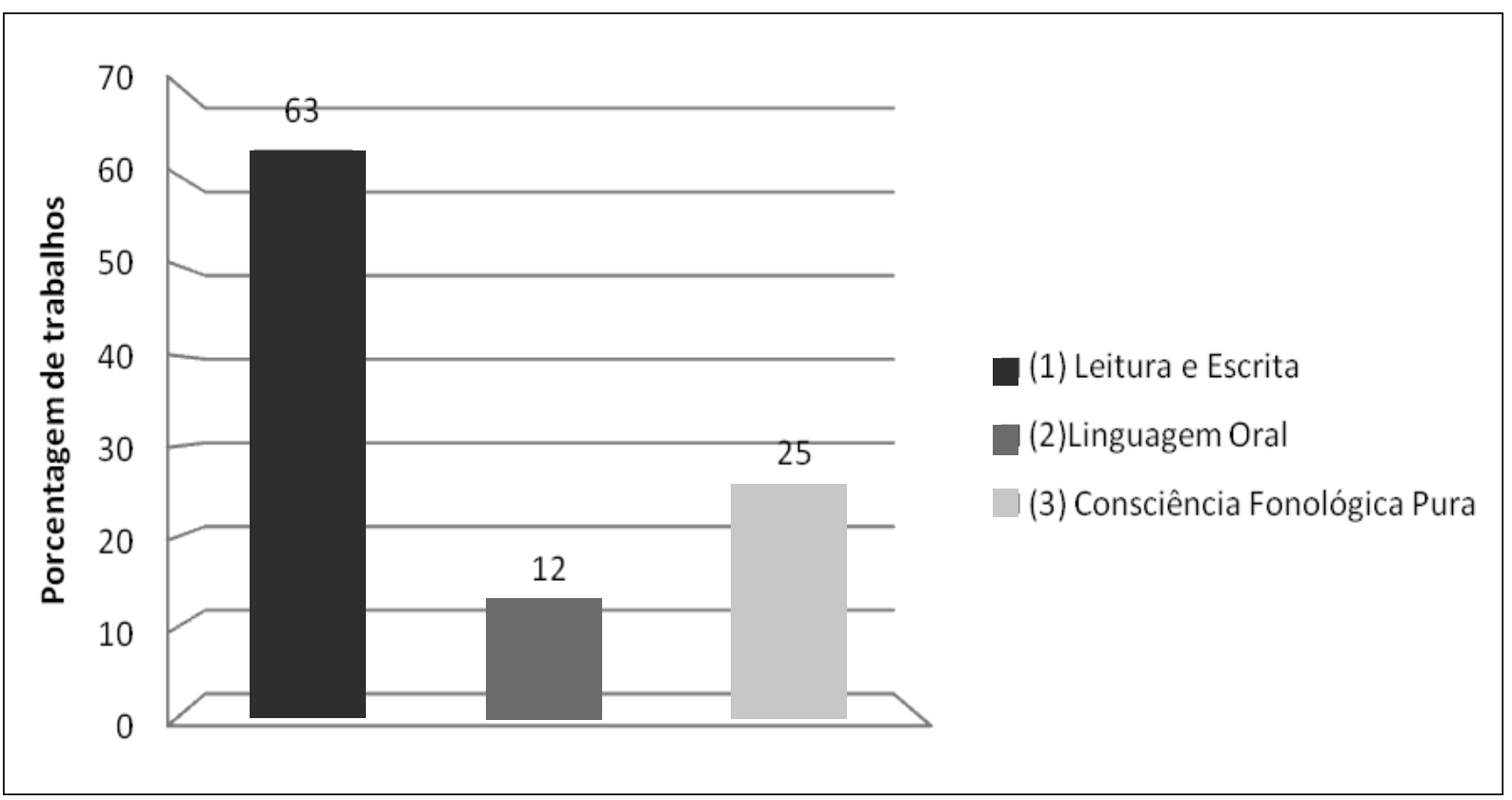

Figura 2. Eixos Temáticos das pesquisas no período entre 2000 a 2010. 


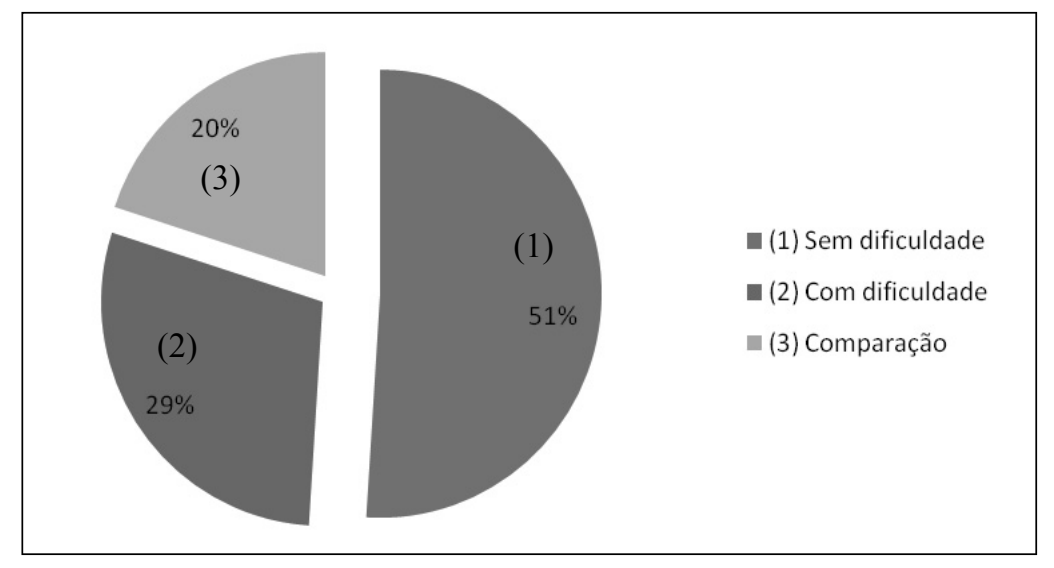

Figura 3. Características da população investigada pelas pesquisas no período 2000 a 2010.

A Figura 3 mostra a distribuição dos trabalhos em relação às características da população investigada. Observa-se que dos 93 trabalhos, 47 (51\%), se dedicaram a investigar sujeitos sem dificuldade; 27 (29\%) estudaram população com dificuldade e 19 trabalhos (20\%), compararam população com e sem dificuldade $(\mathrm{Mo}=1)$.

Com relação ao tipo de população investigada, os resultados mostraram que dos 93 trabalhos, apenas dois trabalhos ${ }^{4}$ foram realizados com adultos $(2,2 \%)$ e outros três trabalhos compararam a população de crianças com a de adultos (3\%). Dessa forma, $95 \%$ dos trabalhos se dedicaram a investigar a consciência fonológica em população de crianças $(\mathrm{Mo}=1)$.

Consideramos que as pesquisas nacionais nesse período podem ter se dedicado especialmente a investigar sujeitos sem queixa de dificuldades como forma de buscar parâmetros de desempenho das habilidades de consciência fonológica. O estabelecimento de parâmetros típicos poderiam ser úteis para a comparação posterior com sujeitos que apresentassem dificuldades de aprendizagem, ou mesmo poderiam auxiliar na busca de respostas aos questionamentos acerca do processo de aquisição da leitura e escrita nos estágios iniciais de alfabetização. Entretanto, considerando a diversidade de testes e tare- fas observada na amostra, tais parâmetros ficam inalcançáveis.

Com relação à faixa de escolarização (Figura 4), 14 trabalhos (15\%) investigaram população de pré-escolares; 11 (12\%), de pré-escola a $4^{\mathrm{a}}$ série; $46(50 \%)$ investigaram população de $1^{\mathrm{a}}$ a $4^{\mathrm{a}}$ série; $13(14 \%)$ de $1^{\mathrm{a}}$ a $8^{\mathrm{a}}$ série e 2 traba$\operatorname{lhos}^{5}(2 \%)$ estudaram a população de EJA- $1^{\mathrm{a}}$ a $4^{\mathrm{a}}$ série $(M d n=3, \mathrm{IQQ}=1)$. Na amostra, 7 trabalhos (7\%) não especificaram qual a faixa de escolarização dos sujeitos investigados. Podemos observar que a porcentagem acumulada nas faixas de escolarização "pré-escolar", "pré-escolar a $4^{\mathrm{a}}$ série" e " $1^{\mathrm{a}}$ a $4^{\mathrm{a}}$ série", representam $76 \%$ da amostra, o que parece demonstrar o interesse dos pesquisadores em investigar a população em torno dos anos iniciais de alfabetização. Por outro lado, apenas dois trabalhos se dedicaram a investigar a população de adultos em processo de alfabetização (EJA).

Da amostra analisada, encontrou-se um total de 15 estudos longitudinais, sendo que dois deles eram longitudinais e de treinamento e um era longitudinal e transversal. Os resultados estão expressos na Tabela 3.
4 Estes dois trabalhos que investigaram apenas a população de adulto, não especificaram qual a faixa de escolarização dos mesmos.
5 Estes dois trabalhos compararam a população de adulto (EJA) com crianças de $1^{\mathrm{a}}$ a $4^{\mathrm{a}}$ série. 


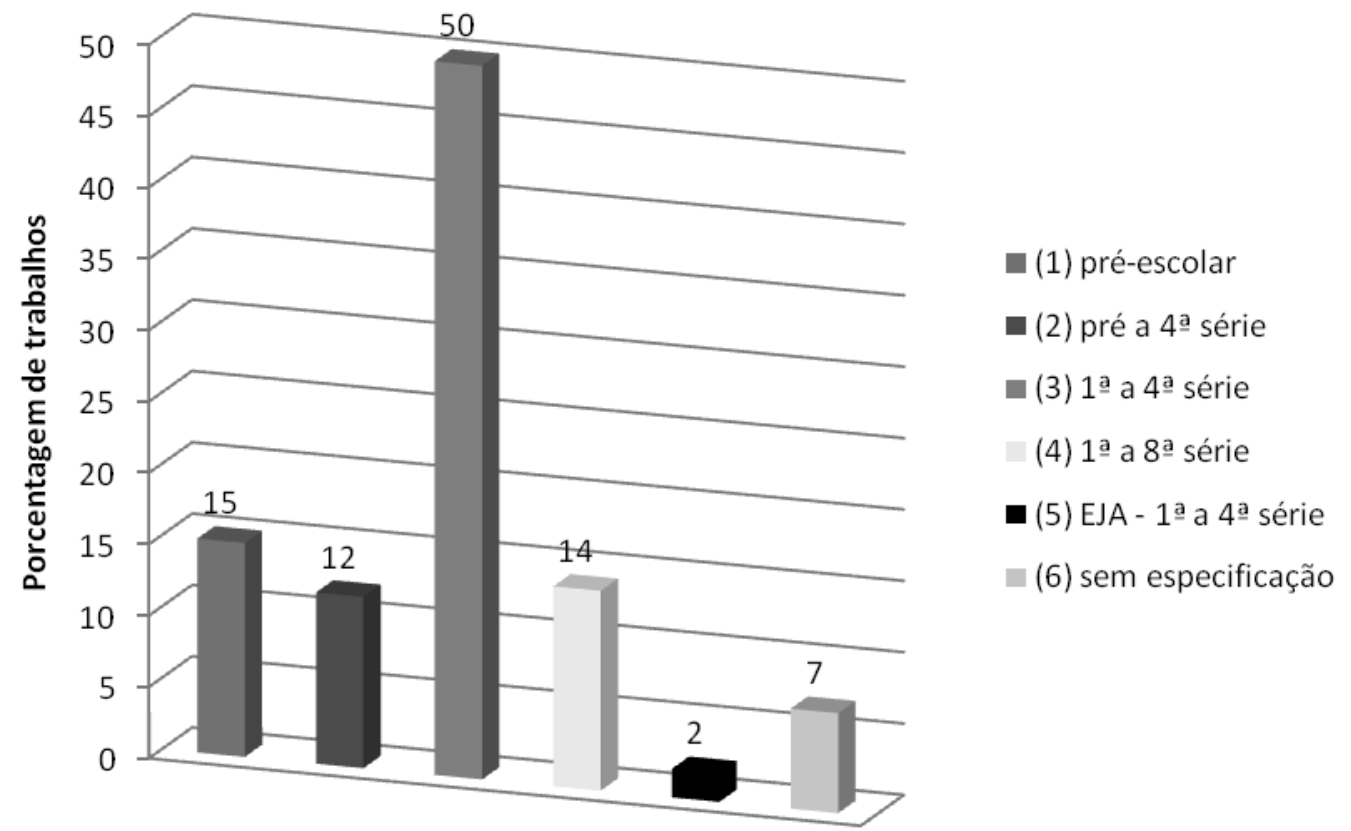

Figura 4. Faixa de escolarização da população investigada pelas pesquisas no período de 2000 a 2010.

Tabela 3

Resultados Qualitativos dos Estudos Longitudinais

\begin{tabular}{lc}
\hline Categoria & Quantidade \\
\hline "não há relação" & 0 \\
"há relação" & 5 \\
"há relação recíproca" & 3 \\
"há relação direta e/ou preditiva" & 3 \\
"não estudou a relação" & 4 \\
\hline Total & 15 \\
\hline
\end{tabular}

Nota. Os valores indicam a qualidade da relação entre consciência fonológica e aprendizagem da leitura expressa pelos resultados de estudos longitudinais. Fonte: dados da pesquisa.

Observou-se que apenas três estudos longitudinais foram categóricos em afirmar a "relação direta e/ou preditiva" das habilidades fonológicas sobre a aprendizagem da leitura e da escrita. Desses, dois afirmaram claramente que a consciência fonêmica é o fator preditivo das habilidades de leitura e de escrita no início da aprendizagem escolar. Como mencionado, para testar a hipótese causal entre duas habilidades correlacionadas, é necessário observar como essas habilidades se articulam no curso do desenvolvimento e realizar o controle de variáveis dependentes sobre a variável independente. Esse tipo de estudo requer desenho metodológico e análise estatística específica o que, supomos, alguns pesquisadores não tiveram como meta principal em suas pesquisas e, por isso, não puderam corroborar resultados mais incisivos a respeito da influência da consciência fonológica. Tais pesquisas reportaram resultados apenas como "há relação" ou "há relação recíproca".

Os estudos de treinamento foram em número de 15, dos quais 12 afirmaram que após o treino da consciência fonológica, houve melhora na aprendizagem da leitura e da escrita, ao passo que três não discorrem sobre a relação causal entre essas habilidades. Observa-se nesses resultados a confirmação dos principais resultados descritos na literatura internacional, ao reafirmar a importância das habilidades fonológicas para o processo de aprendizagem.

Finalmente, buscou-se evidências a respeito da contribuição da consciência fonológica aos casos de sujeitos com dificuldades de aprendizagem. Das 125 pesquisas, entre teses e dissertações, foram encontrados 17 trabalhos que tratavam desse tipo de população. Destes, oito trabalhos investigaram população denominada pelo pesquisador como disléxica e os outros nove trabalhos investigaram população denomi- 
nada como sendo distúrbio ou dificuldade para aprender a ler e/ou a escrever. Dos trabalhos com sujeitos disléxicos, apenas quatro relacionaram e investigaram a consciência fonológica como causa das dificuldades em leitura. Os resultados reportados afirmaram, em três desses trabalhos, ter encontrado um prejuízo nas habilidades de consciência fonológica dos disléxicos, o que está em linha com os resultados internacionais. O quarto trabalho afirmou que o treino em consciência fonológica favoreceu o domínio das habilidades de leitura e escrita entre os disléxicos.

Sabendo-se que a literatura internacional aponta como principal causa da dislexia o prejuízo nas habilidades fonológicas, consideramos que o número de pesquisas brasileiras, as quais têm se dedicado a compreender o fenômeno relacionando-o às descobertas mais atuais da ciência da leitura, ainda é pequeno e a temática precisa ser aprofundada, sobretudo quando se tem em cena a discussão com relação à influência das características da ortografia (opaca/transparente) que se aprende.

A análise dos resultados dos demais trabalhos que investigaram população com distúrbio ou dificuldade para aprender a ler e/ou a escrever, mostrou que seis deles evidenciaram que ao treinar a consciência fonológica, consequentemente se obtém a melhora do aprendizado e este treino contribui para amenizar a dificuldade que havia anteriormente. Os três trabalhos restantes não tinham como foco relacionar a consciência fonológica à dificuldade ou distúrbio.

Esses resultados corroboram, ainda que timidamente, a contribuição específica da consciência fonológica para a reabilitação das dificuldades de aprendizagem da leitura e da escrita e seu papel crítico para a aprendizagem alfabética.

\section{Discussão}

As habilidades metalinguísticas se configuram em um conjunto de habilidades que tem sido apontado como diretamente implicado no processo de aprendizagem da leitura e da escrita. Algumas habilidades metalinguísticas, como a consciência morfossintática e a consciência metatextual, têm sido alvo de recentes pesquisas na área, entretanto, ainda são pouco conhecidas suas implicações para o processo de aprendizagem alfabética. Já as pesquisas com relação à contribuição da consciência fonológica têm fortemente evidenciado a importância da consciência fonêmica como uma habilidade estreitamente relacionada ao sucesso da leitura e da escrita e como principal causa da dislexia. Nesse cenário, compreender como esta habilidade participa do processo de alfabetização, como ela pode estar presente nas atividades do cotidiano escolar, ou mesmo como lançar mão desse recurso para a reabilitação das dificuldades de aprendizagem, passou a ser foco de muitas pesquisas.

Este artigo objetivou desenhar um panorama das pesquisas nacionais que no período entre 2000 e 2010 investigaram as habilidades de consciência fonológica com a aplicação de testes. Para isso foram identificados: tipo de pesquisa (mestrado/doutorado) e ano de publicação, área de conhecimento da formação inicial e em nível de pós-graduação dos pesquisadores, quais testes de consciência fonológica têm sido utilizados pelos pesquisadores brasileiros, a temática de maior predominância nas pesquisas, o tipo, as características e a faixa de escolarização da população investigada. Além disso, deu-se especial atenção aos estudos longitudinais, de treinamento e com sujeitos com dificuldades de aprendizagem, com o intuito de evidenciar os principais resultados sobre a contribuição das habilidades de consciência fonológica ao processo de alfabetização.

Como resultado, de maneira geral, observou-se que a pesquisa nacional nesta área encontra-se em desenvolvimento, já não em estágio inicial, mas necessita ainda de atenção por parte dos pesquisadores. Registramos pequeno número de pesquisas em nível de doutorado. As pesquisas no período entre 2000 e 2010 tiveram como foco investigar a temática relacionada ao desenvolvimento/dificuldades da leitura e da escrita em população de crianças sem queixas de dificuldades de aprendizagem e que se encontravam no período inicial de aquisição da linguagem escrita. A partir disso, sugere-se a ampliação da pesquisa no campo da caracterização da importância da consciência fonológica aos pro- 
cessos de aprendizagem alfabética em relação à população de jovens e adultos, bem como a comparação entre populações com e sem queixa de dificuldades de aprendizagem.

Apesar das pesquisas terem priorizado a população de crianças em fase inicial de aprendizagem alfabética, em função de não haver convergência quanto ao uso de instrumentos de avaliação da consciência fonológica pelos pesquisadores, tornou-se difícil afirmar como as habilidades de consciência fonológica se desenvolvem no curso da experiência linguística e/ou de aquisição da linguagem escrita no português do Brasil. Ressaltamos que muitos trabalhos carecem de rigor metodológico e não trazem informação suficiente para que se possa proceder a uma metanálise. Como característica geral, percebemos também que grande parte dos trabalhos se dedicaram a estudar um número pequeno da população alvo, o que impediu a generalização de resultados. Aliada a estas questões, a diversidade e fragilidade de muitos testes e tarefas de consciência fonológica, produzem resultados pouco confiáveis.

Entendemos que é necessária a validação de um teste de consciência fonológica para avaliar de forma confiável os diferentes níveis dessa habilidade e fornecer ao pesquisador, como também ao professor e ao terapeuta, parâmetros para identificar as habilidades que podem ser estimuladas durante a educação infantil e nos anos iniciais de escolarização. Com isso será possível desenvolver estratégias e programas de prevenção e/ou reabilitação de dificuldades de aprendizagem e/ou favorecer o processo de aquisição alfabética.

Para que fosse corroborada a relação causal entre consciência fonológica e aprendizado da leitura e da escrita, tomou-se como foco os estudos longitudinais e de treinamento. De forma surpreendente observou-se que a maioria dos estudos longitudinais não se deteve em estabelecer esta relação causal. O construto "consciência fonológica" é unânime, na atualidade, em admitir uma relação recíproca entre essas duas habilidades, por isso, interessa saber qual a real influência das habilidades de consciência fonológica, em seus diferentes níveis, sobre a aprendizagem de diferentes ortografias alfabéticas (transparentes e opacas). No Brasil, esse aspecto da pesquisa ainda é embrionária. Com isso, os principais resultados advêm dos estudos de treinamento ao afirmarem que o treino das habilidades de consciência fonológica melhora o aprendizado ou ameniza a dificuldade.

Os estudos com sujeitos disléxicos foram poucos, e desses, menor número ainda se dedicou a investigar a relação dessa dificuldade com a consciência fonológica. Nesse sentido, os resultados do presente estudo apontam para a necessidade de maior comprovação da contribuição específica das habilidades de consciência fonológica como causa da dislexia, sobretudo na ortografia do português do Brasil, que tem características de maior transparência para a leitura. A compreensão da causa da dislexia poderia auxiliar sobremaneira a atividade pedagógica, auxiliando o professor na escolha de metodologias mais eficientes e ajudar na condução das dificuldades tanto no ambiente escolar como na clínica de reabilitação. Sugere-se, portanto, pesquisas com a avaliação da consciência fonológica, especialmente de nível fonêmico, em sujeitos disléxicos.

Sugere-se também maior investigação com relação à contribuição da consciência fonológica para a aprendizagem especialmente dos processos de escrita, em função de suas características de maior opacidade em relação à leitura, no português do Brasil.

Por outro lado, os resultados alcançados por alguns estudos longitudinais e pelos estudos de treinamento confirmam os principais resultados das pesquisas internacionais: a consciência fonológica, no nível fonêmico, é um forte preditor de sucesso das habilidades de leitura e de escrita, e a estimulação dessas habilidades facilita esta aprendizagem. Nesse sentido, recomenda-se a ampliação de programas de estimulação das habilidades de consciência fonológica na educação infantil e nos anos iniciais de escolarização, com o intuito de prevenir e facilitar a aquisição alfabética. A estimulação da consciência fonológica deve ganhar espaço na sala de aula através de jogos e brincadeiras (como fonte de consulta, sugerimos Adams, Foorman, Lundberg, \& Bee- 
ler, 2006). Para tanto, é necessário que o professor compreenda a vinculação dessas habilidades no curso do desenvolvimento de linguagem dos pequenos, mas sobretudo, compreenda que, nos anos iniciais de escolarização, a consciência fonêmica, como último e mais importante nível das habilidades de consciência fonológica, se desenvolve com o ensino das correspondências grafema-fonema. Será a partir da mediação do professor que as crianças (e também os jovens e adultos) em processo inicial de alfabetização poderão desenvolver a atenção e identificar o fonema na cadeia contínua da fala, para então, a ele, atribuir um grafema. Essa descoberta implica, como assinala Morais (1996), no aspecto crítico da alfabetização.

\section{Considerações Finais}

O corpo de resultados nos permite afirmar que na última década (2000-2010) os pesquisadores brasileiros que se utilizaram de testes de consciência fonológica em seus estudos tiveram como foco investigar a relação das habilidades de consciência fonológica com a aprendizagem da linguagem escrita em sujeitos sem queixas de dificuldades de aprendizagem durante o processo inicial de alfabetização. Os profissionais que se dedicaram a essas investigações, prioritariamente em nível de mestrado, advêm principalmente das áreas clínicas, seguidos, em menor número, dos profissionais da educação.

Uma vez que a investigação do construto "consciência fonológica" é bastante amplo, verificou-se que o principal resultado das pesquisas brasileiras que buscaram compreender a relação causal entre as habilidades de consciência fonológica e a aprendizagem alfabética, caracterizadas por estudos longitudinais e de treinamento, ainda que em pequeno número, evidenciou a correlação entre essas habilidades, mas também ressaltou o caráter preditivo das habilidades de consciência fonológica sobre a aprendizagem da leitura e da escrita, como também demonstrou que o treino dessas habilidades melhora a aprendizagem alfabética. Por fim, constatou-se, nos poucos estudos que investigaram a natureza das dificuldades de aprendizagem, que um pre- juízo em consciência fonológica está associado à dislexia.

\section{Referências}

Adams, M. J., Foorman, B. R., Lundberg, I., \& Beeler, T. (2006). Consciência fonológica em crianças pequenas (R. C. Costa, trad.). Porto Alegre, RS: Artmed.

Alegria, J., Leybaert, J., \& Mousty, P. (1997). Aquisição da leitura e distúrbios associados: Avaliação, tratamento e teoria. In J. Grégoire \& B. Piérart (Eds.), Avaliação dos problemas de leitura: Os novos modelos teóricos e suas implicações diagnósticas (pp. 105-124). Porto Alegre, RS: Artes Médicas.

Alencar, M. L. (2000). Desenvolvimento e desempenho cognitivo: Construção e validade de uma bateria de habilidades cognitivas para as crianças de Estado do Ceará (Tese de doutorado em Educação e Psicologia, Universidade do Minho, Braga, Portugal).

Alvarez, A. M., Carvalho, I. A., \& Caetano, A. L. (1998). Perfil de habilidades fonológicas - Manual. São Paulo, SP: Via Lettera.

Alvarez, A. M. M. A., Carvalho I. A. M., \& Caetano, A. L. (2004). Perfil de habilidades fonológicas (2. ed.) São Paulo, SP: Via Lettera.

Aquino S. B. (2007). O trabalho com consciência fonológica na educação infantil e o processo de apropriação da escrita pelas crianças (Dissertação de mestrado em Educação, Universidade Federal de Pernambuco, Ceará, PE, Brasil).

Aro, M., \& Wimmer, H. (2003). Learning to read: English in comparison to six more regular orthographies. Applied Psycholinguistics, 24, 621635. doi:10.1017.S0142716403000316

Barrera, S. D. (2000). Linguagem oral e alfabetização: Um estudo sobre variação lingüística $e$ consciência metalingüística em crianças da $1^{a}$. série do ensino fundamental (Tese de doutorado em Psicologia, Universidade de São Paulo, SP, Brasil).

Bertelson, P., \& Gelder, B. (1991). The emergence of phonological awareness: Comparative approaches. In I. G. Mattingly \& M. StuddertKennedy (Eds.), Modularity and the motor theory of speech perception: Proceedings of a conference to Honor Alvin M. Liberman (pp. 393-412). New York: Lawrence Erlbaum. 
Bradley, L., \& Bryant, P. E. (1983). Categorizing sounds and learning to read: A casual connection. Nature, 301, 419-421. doi:10.1038/301419a0

Capellini, S. A., \& Smythe, I. (2008). Protocolo de avaliação de habilidades cognitivo-linguísticas: Livro do profissional e do professor. Marília, SP: Fundação para o Desenvolvimento do Ensino, Pesquisa e Extensão.

Capovilla, A. G. S., \& Capovilla, F. C. (1998). Prova de consciência fonológica: Desenvolvimento de dez habilidades da pré-escola à segunda série. Temas sobre Desenvolvimento, 7, 14-20.

Capovilla, A. G. S., \& Capovilla, F. C. (2000). Problemas de leitura e escrita: Como identificar, prevenir e remediar numa abordagem fônica. São Paulo, SP: Memnon.

Capovilla, A. G. S., Capovilla, F. C., \& Silveira, F. B. (1998). O desenvolvimento da consciência fonológica, correlações com leitura e escrita e tabelas de normatização. Ciência Cognitiva, Teoria, Pesquisa e Aplicação, 2, 113-160.

Cardoso-Martins, C. (1991). A sensibilidade fonológica e a aprendizagem inicial da leitura e da escrita. Cadernos de Pesquisa, 76, 41-49.

Cardoso-Martins, C. (1995). Sensitivity to rhymes, syllables, and phonemes in literacy acquisition in Portuguese. Reading Research Quaterly, 30, 808-828.

Carraher, T. N., \& Rego, L. L. B. (1981). O realismo nominal como obstáculo na aprendizagem da leitura. Cadernos de Pesquisa, 39, 3-10.

Castles, A., \& Coltheart, M. (2004). Is there a causal link from phonological awareness to success in learning to read? Cognition, 91, 77-111. doi:10.1016/S0010-0277(03)00164-1

Cielo, C. A. (2002). Habilidades em consciência fonológica em crianças de 04 a 08 anos de idade. Pró-Fono, 14, 287-478.

Corrêa, M. F. (2009). Como os adultos aprendem a ler? Evidências de um estudo com adultos pouco alfabetizados e crianças com a mesma habilidade de leitura (Dissertação de Mestrado em Psicologia, Universidade Federal de Minas Gerais, Belo Horizonte, MG, Brasil).

Defior, S. (2004). Phonological awareness and learning to read: A cross-linguistic perspective. In T. Nunes \& P. Bryant (Eds.), Handbook of children's literacy (pp. 631-649). London: Kluwer.
Defior, S., Martos, F., \& Cary, L. (2002). Differences in reading acquisition development in two shallow orthographies: Portuguese and Spanish. Applied Psycholinguistics, 23, 135-148. doi:10.1017.S0142716402000073

Defior, S., \& Tudela, P. (1994). Effect of phonological training on reading and writing acquisition. Reading and Writing, 6, 299-320.

Diniz, N. L. B. (2008). Metalinguagem e efeitos de uma intervenção para recuperação de alunos com dificuldade na aprendizagem da linguagem escrita (Tese de doutorado em Psicologia, Universidade de São Paulo, SP, Brasil).

Fernandes, F., Andrade, C. R. F., Befi-Lopes, D. M., \& Wertzner, H. F. (1998). Manual de avaliação da linguagem do Centro de Saúde Escola Samuel B. Pessoa. São Paulo, SP: Centro de Saúde Escola Samuel B. Pessoa.

Godoy, D. M. A. (2001). Testes de consciência fonológica e suas relações com a aprendizagem da leitura no Português (Dissertação de mestrado em Linguística, Universidade Federal de Santa Catarina, Florianópolis, SC, Brasil).

Godoy, D. M. A., Koglin, A., \& Abreu, G. S. A. (2011). Interferência de representações ortográficas à execução de tarefas de consciência fonológica no processo de alfabetização. Trabalho apresentado no XVI Congreso Internacional de la Asociación de Linguistica y Filologia de la America Latina - ALFAL, Madrid, Espanha.

Guedes, M. C. R. G. (2009). Consciência fonológica em periodos pré e pós-alfabetização (Dissertação de mestrado em Linguística, Universidade Federal do Rio de Janeiro, RJ, Brasil).

Hatcher, P. (1994). Sound linkage: An integrated programme for overcoming reading difficulties. London: Whurr s.

Herrero, S. F. (2001). Perfil das crianças pré-escolares e escolares no Teste de Sensibilidade Fonológica (Dissertação de Mestrado em Linguística, Universidade de São Paulo, SP, Brasil).

Ilha, S. E. (2004). A aquisição da estrutura silábica na escrita inicial de crianças e adultos: Uma relação com a consciência fonológica (Tese de doutorado em Letras, Pontifícia Universidade Católica do Rio Grande do Sul, Porto Alegre, RS, Brasil).

Jong, P. F. (2003). Problems in the acquisition of fluent word decoding in Dutch children. In N. Goulandris (Ed.), Dyslexia in different languages: 
Cross linguistic comparisons (pp. 33-52). London: Whurr.

Jong, P. F., \& Van Der Leij, A. (1999). Specific contributions of phonological abilities to early reading acquisition: Results from a Dutch latent variable longitudinal study. Journal of Educational Psychology, 91, 450-476. doi:10.1037/00220663.91.3.450

Landerl, K., WImmer, H., \& Frith, U. (1997). The impact of orthographic consistency on dyslexia: A German-English comparison. Cognition, 63, 315-334. doi:10.1016/S0010-0277(97)00005-X

Liberman, I. Y., Shankweiler, D., Fisher, F. W., \& Carter, B. (1974). Explicit syllable and phoneme segmentation in the young child. Journal of Experimental Child Psychology, 18, 201-212.

Lindamood, C. H., \& Lindamood, P. C. (1979). The LAV Test - Lindamood Auditory Conceptualization Test. $\left(2^{\text {nd }}\right.$ ed.). Austin, TX: Pro-Ed.

Lundberg, I., Frost, J., \& Petersen, O. (1988). Effects of an extensive program for stimulating phonological awareness in preschool children. Reading Research Quaterly, 23, 263-284.

Maluf, M. R., Pagnez, K. S. M., \& Zanella, M. S. (2006). Habilidades metalingüísticas e linguagem escrita nas pesquisas brasileiras. Boletim de Psicologia, 124, 67-92.

Moojen, S., Lamprecht, R., Santos, R. M., Freitas, G. M., Brodacz, R., Siqueira, M., ...Guarda, E. (2003). Consciência fonológica: Instrumento de avaliação seqüencial. São Paulo, SP: Casa do Psicólogo.

Morais, J. (1996). A arte de ler. São Paulo, SP: Editora da Universidade Estadual Paulista "Júlio de Mesquita Filho".

Morais, J., Cary, L., Alegria, J., \& Bertelson, P. (1979). Does awareness of speech as a sequence of phones arise spontaneously? Cognition, 7, 323-331.

Morais, J., Bertelson, P., Cary, L., \& Alegria J. (1986). Literacy training and speech segmentation. Cognition, 24, 45-64. doi:10.1016/00100277(86)90004-1

Mousty, P., Leybaert, J., Alegria, J., Content, A., \& Morais, J. (1994). Belec - une batterie d'évaluation du langage écrit et de ses troubles. In J. Grégoire \& B. Piérart (Eds.), Evaluer les troubles de la lecture (pp. 127-145). Bruxelles, Belgique: De Boeck-Wesmael.
Pacheco, C. B. (2009). Habilidades musicais e consciência fonológica: Um estudo correlacional com crianças de 4 a 5 anos de Curitiba (Dissertação de mestrado em Música, Universidade Federal do Paraná, Curitiba, PR, Brasil).

Porpodas, C. D. (1991). The relation between phonemic awareness and reading and spelling of Greek words in the first school years. In M. Carrero, M. Pope, R.-J. Simons, \& J. I. Pozo (Eds.), Learning and instruction: European research in an international context (pp. 203-217). Oxford, UK: Pergamon Press.

Rack, J. P., Snowling, M. J., \& Olson, R. (1992). The nonword reading deficit in developmental dyslexia: A review. Reading Research Quarterly, 19, 278-301.

Rayner, K., \& Pollatsek, A. (1989). The psychology of reading. London: Prentice-Hall International.

Read, C., Zhanc, Y., Nie, H., \& Dincg, B. (1986). The ability to manipulate speech sounds depends on knowing alphabetic reading. Cognition, 24, 31-44.

Santos, A. (1996). A influência da consciência fonológica na aquisição da leitura e da escrita. In F. Sisto, G. Oliveira, L. Fini, M. Souza, \& R. Brenelli (Eds.), Atuação psicopedagógica e aprendizagem escolar (pp. 213-247) Petrópolis, RJ: Vozes.

Santos, M. T. M., \& Pereira L. D. (1997). Consciência fonológica. In L. D. Pereira \& E. Schochat (Eds.), Processamento auditivo central: Manual de avaliação (pp. 198-196). São Paulo, SP: Lovise.

Scliar-Cabral, L. (2003). Princípios do sistema alfabético do português do Brasil. São Paulo, SP: Contexto.

Serrano, F., \& Defior, S. (2008). Dyslexia speed problems in a transparent orthography. Annals of Dyslexia, 58, 81-95. doi:10.1007/s11881008-0013-6

Siccherino, L. A. F. (2007). Consciência fonológica e aquisição da escrita: Um estudo com crianças do $1^{\circ}$ ano do Ensino Fundamental (Dissertação de mestrado em Educação, Pontifícia Universidade Católica de São Paulo, SP, Brasil).

Snowling, M. (1998). Dyslexia as a phonological deficit: Evidence and implications. Child Psychology and Psychiatry Review, 3, 4-11.

Stanovich, K. E., Cunningham, A. E., \& Cramer, B. B. (1984). Assessing phonological awareness in 
kindergarten children: Issues of task comparability. Journal of Experimental Child Psychology, 38, 175-190.

Vaessen, A. A., \& Blomert, L. (2010). Long-term cognitive dynamics of fluent reading development. Journal of Experimental Child Psychology, 105, 213-321. doi:10.1016/j.jecp.2009.11.005

Wagner, R., Torgesen, J., \& Rashotte, C. (1999). Comprehensive Test of Phonological Processing (CTOPP). Austin, TX: Pro-Ed.

Wimmer, H., \& Goswami, U. (1994). The influence of orthographic on reading development: Word recognition in English and German children. Cognition, 51, 91-103.
Wimmer, H., Landerl, K., Linortner, R., \& Hummer, P. (1991). The relationship of phonemic awareness to reading acquisition: More consequence than precondition but still important. Cognition, 40, 219-249. doi:10.1016/0010-0277(91)90026-Z
Recebido: 23/05/2013

$1^{a}$ revisão: 6/09/2013

$2^{a}$ revisão: $4 / 12 / 2013$

Aceite final: 4/12/2013 